\title{
COVID-19: Combating the Pandemic One Year on - Street Wise or Street Science?
}

\author{
U. Tuzun* \\ Alumnus Professor, University of Cambridge, UK \\ Professional Consultant, Weybridge, Surrey, UK
}

Submission: December 12, 2020; Published: January 26, 2020

*Corresponding author: U. Tuzun, Alumnus Professor, University of Cambridge, UK, Professional Consultant, Weybridge, Surrey, UK

\begin{abstract}
The article will probe the measures implemented on public irrespective of age group, gender and other social markers developed in the earlier article which are aimed at "catching and isolating" positive testers as early as possible whilst the preventative practices remain broadly street based, i.e. wash-mask-no contact. The street science that governs these implementations rely on the statistical paradigm that if the human to human spreading is not kept below the reproduction rate factor of $R=1$, then the positive cases will continue to multiply in geometric progression resulting an exponential increase in hospitalization cases. There appears to be no independent scientific check of these hypotheses accept by the accumulation of statistical data sets of the increase in positive test results which show significant variations in regions and countries as the number of tests carried out multiply also in geometric progression. In the meantime, minimal laboratory science information is released to general public whilst a significant number of vaccine trials across the world are held and with some already registered as officially approved by different governments. Given the availability of high volumes of test and trace data available globally and the very large numbers of vaccine trials being held across the globe, what is preventing the curtailment of the spread of the virus? A tentative framework is presented based on neural network constructions of the connectivity of the laboratory and demographic (street- based) scientific data sets that could explain the apparent lack of rapid progress in combating the spread of COVID-19 almost one year on.
\end{abstract}

Keywords: Positive testers; Geometric progression; COVID-19; Psychology; Behaviour; Covid-19 pandemic.

\section{Topological Psychology (Gestalt Psychologie) and its} Application to Spread of Pandemic

Kurt Lewin [1,2] has introduced his field theory of psychology to the social psychology literature in the States in early $20^{\text {th }}$ century following from the German and Austrian school of Gestalt Psychologie principles of proximity, similarity, continuity, connectedness and collectiveness to describe in a holistic manner the patterns of interactions between the individuals in a human society and their interactions with their environment. Lewin's celebrated equation of field psychology is expressed most simply in the form:-

$$
B=f(P, E)
$$

Here, B stands for human behavior, $\mathrm{P}$ is for person (individual) and $\mathrm{E}$ denotes their environment. This simply states that psychological behaviour is a function of the person and their environment. It is possible to extend this visionary approach further to a neural network formulation as follows:

$$
\begin{aligned}
& B(s, t)=f(P(s, t), E(s, t)) \\
& C(s, t)=f\left(B(s, t), E^{\prime}(s, t)\right)
\end{aligned}
$$

The environment $\mathrm{E}(\mathrm{s}, \mathrm{t})$ is a dynamically evolving parameter whose precise state is defined at a given space, $s$ and time, $t$. Human behaviour, B is likewise dynamically evolving with space and time. Hence, we can now introduce another dynamically evolving parameter, $\mathrm{C}(\mathrm{s}, \mathrm{t})$ to represent the consequence as a function of the human behavior, $\mathrm{B}(\mathrm{s}, \mathrm{t})$ and the altered state, $\mathrm{E}^{\prime}$ $(\mathrm{s}, \mathrm{t})$ of the environment. With this simple first order functionality of interactions between the individual, community and the socioeconomic environment is amply accounted for. Furthermore, it is possible to extend this neural network analysis to higher order interactions by recognizing and incorporating subsets of variables 
defining various personality attributes, physical condition, age, gender and ethnicity of the individual as well as environmental attributes such as education, occupation contributing to the health, wealth and wellbeing of the individual. The author has demonstrated the applicability of the connectivity of these factors in student-centered learning in higher education and in life-long learning [4] as well as in modelling the connectivity in human behavioral system of surrounding systems affecting Covid-19 immunity; [3].

\section{Modelling Connectivity of Population Samples using Large Demographic Data Sets}

The inclusion of the subsets of variables describing the statistical distribution of personal attributes and environmental factors into the dynamically evolving field theory above requires statistical modelling of the spatial and temporal variations of these parameters and thus resulting in mathematical expressions that represent the statistics of the dynamic evolution of the local fields at different times by quantifying the effects (consequences) of human behavior on the environment and vice-versa. The recent work by Eichenbaum et al. [5] precisely follows this route to investigate the Covid-19 infection risk perception of population samples of age groups of using large data sets (typically $500 \mathrm{k}$ individuals) randomly selected from the 6.3 million population statistics produced by Statistics Portugal between January 2018May 2020). These statistical datasets include income levels, occupation and spending patterns of individuals, age, sex and comorbidity data relating to underlying health conditions such as heart problems, cancer, obesity and type- 2 diabetes; refer also to [5], and Dowd et al. [6] who reported statistical correlations of Covid-19 fatality rates of populations in different countries such as South Korea and Italy. These two very recent studies represent good examples of the dynamic field theory implementation.

Eichenbaum et al. [5] model the connectivity functions between modified human behaviour as a function of personal data sets and environmental factors. Perception of possible risk of infection by young and elderly population groups is demonstrated to be significantly different by monitoring their acquisition of services and goods requiring frequent social contact with those requiring low-contact acquisitions based on the digital datasets available in government and telecommunication databases of income levels, expenditure and tax details. Large differentials are found between the young and elderly age groups in that those with high income and assets and secure employment tend to isolate more readily and avoid acquisitions requiring frequent social contact and use of transportation. This more effective socioeconomic operant behaviour modification due to heightened perception of Covid-19 infection risk among the rich and the elderly demonstrates the first order interactions between the individual behaviour and the environmental factors expressed in eqns. (1-3) above. Perhaps, much more surprisingly, the individual's comorbidity (underlying chronic health conditions) has much less of an impact on modification of operant social behaviour to avoid and/or minimise risk of infection compared to the effectiveness of socio-economic habitat factors. The comorbidity condition is found to lead to somewhat higher infection risk awareness and socio-economic modification behaviour among the elderly age groups compared to the young groups. It appears that those who stand to lose much due to the spread of infection behave with less infection risk awareness and less practice of modified social behavior requiring protective measures, i.e. mask-wash and nocontact constraints. Outcomes of this inverse connectivity of risk perception and social status with readiness to modify social behavior to contain the virus transmission is abundantly clear in the fatality statistics produced in local populations in the States and elsewhere. Rich and elderly prove to be much more streetwise in avoiding Covid-19 infection.

Dowd et al. [6] report only on observational statistics of large field data sets and use mathematical tools to identify patterns between fatality rates and physical attributes of individual data subsets paying specific attention to demographic distributions of age and sex and the specific social interaction patterns between the young and elderly groups of the national and regional populations in affecting the spread of the virus and the much higher fatality rates among the elderly population groups. Of particular interest in their statistical correlations is the effect of mobility/travel between the young and old population groups and the level of intergenerational social interactions as a function of factors like ethnicity and religion. Communities with high levels of physical mobility and intergenerational social interactions tend to give rise to higher values of the reproduction number, Ro of the virus. The much higher population density of the industrial urban environments often with highly restrictive housing conditions compared to rural, agricultural communities adds to the spread rate of the virus by providing multiple levels of individual and community social interaction and local travel such as visits to local shops, professional offices, educational and catering service providing establishments to name but a few.

\section{Force-Field Analysis of Spread of Covid-19 and Non-Pharmaceutical Interventions for Containment}

Force field analysis is based on a model for thinking about change as proposed by Kurt Lewin, who saw behavior in an institutional setting not as a static (motionless) habit or pattern but as a dynamic balance of forces working in opposite directions Lewin [1,2]. According to this way of looking at patterned behavior, change takes place when an imbalance occurs between the sum of the forces against change (Restraining Forces) and the sum of the forces for change (Driving Forces). A force-field analysis assumes that any social situation is a balance between these forces. An imbalance may occur through a change of magnitude or a change in direction in any one of the forces, or through the addition of a new force. A schematic illustration of the force-field equilibrium is provided in Figure 1. 


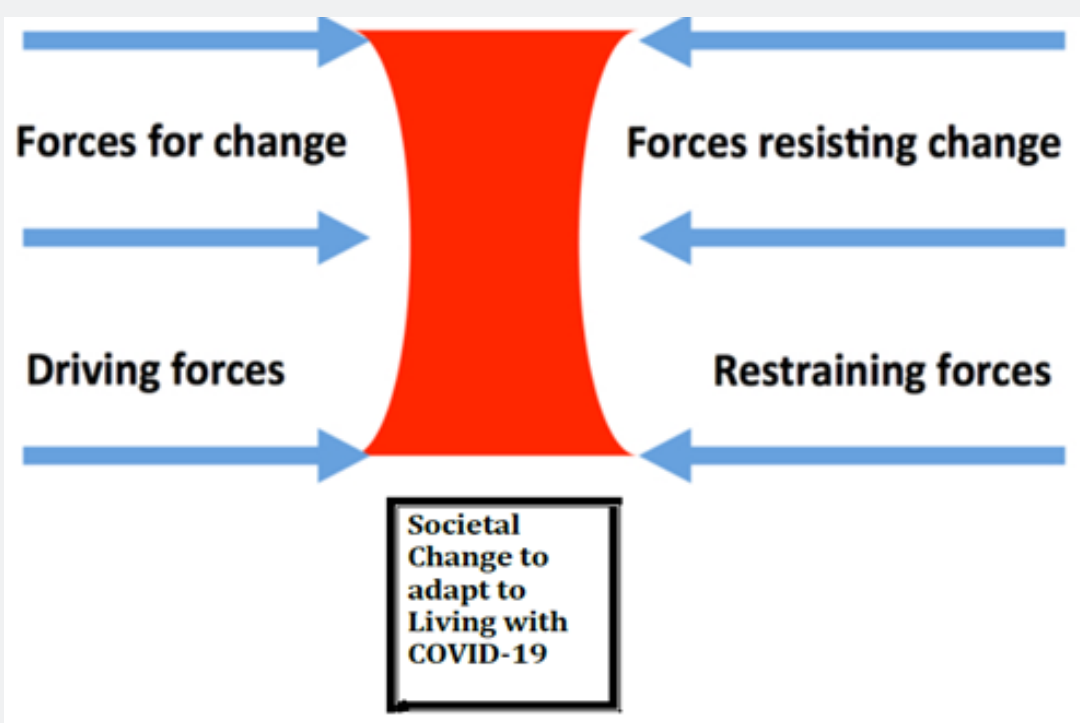

Figure 1: Kurt Lewin's (1943) Force Field Analysis Applied to Societal Change to adapt to Living with COVID-19.

Here it is useful to establish the factors that influence the direction and magnitude of societal change due to COVID-19 under the categories of forces suggested by Lewin. Table 1 provides a possible list of system components for inclusion in the assessment of driving and restraining forces of the spread of the Covid-19 pandemic.

Table 1: Forces Driving and Resisting the Continuation of COVID-19 Pandemic balanced against the Forces for Societal Change and Measures Applied to Restrain the Adverse Effects

\begin{tabular}{|l|l|}
\hline \multicolumn{1}{|c|}{ Forces for Change } & \multicolumn{1}{|c|}{ Restraining Forces } \\
\hline Disruption to Public Services & Income Support Schemes \\
\hline Disruption to Industry Sectors & Tax and Mortgage Postponement \\
\hline Redundancy and Loss of Income & Business and Industry Support \\
\hline Loss of Physical Social Contact & Alternative Employment Schemes \\
\hline Loss of Business Activity Driving Forces & Free Social and Healthcare \\
\hline & Resisting Forces \\
\hline Spread Rates of Infection $\quad$ Health Protective Measures \\
\hline Increasing Number of Hospitalizations & Test \& Trace \\
\hline Increasing Intensity of Infection & Self-Isolation \\
\hline Increasing Mortality Rates & Blood Plasma Donations \\
\hline Slow Progress with Therapeutic Drugs \& Vaccine Development \& Social Care Staff & Increased Experience of Hospital \\
\hline Anticipation of Protracted Vaccine Testing \& Field Trials & Improved Hospital Facilities \\
\hline & Low Reinfection Rates \\
\hline
\end{tabular}

It is important to recognize the action components in Table 1 under the sections of Forces for Change and Restraining Forces as non-pharmaceutical and socio-economic policy interventions whilst the sections of Driving Forces and Resisting Forces respectively to represent the pharmaceutical and medical interventions. The system component action lists are by no means exhaustive and are also subject to change in priority levels in fast evolving emergency scenarios. A further important aspect of the force-field analysis is the rate of information flow between nonpharmaceutical and pharmaceutical/medical data collection activities and their respective statistical evaluation and projection modelling of future trends. After all, the dynamics of the impacts of the pharmaceutical and medical, non-pharmaceutical and socioeconomic measures on the force-field equilibrium depicted in Fig.1 depend highly on the effectiveness of information transfer based on large-data storage readiness and speedy organization of happenstance data into statistical evaluations to assist with decision-making in emergency scenarios. A recent Royal Society DELVE Initiative report [7] recommends that happenstance data pertaining to individual citizens and their daily activities need to 
be i) anonymized, ii) aggregated and iii) statistically calibrated to arrive at meaningful metrics for robust and rapid decisionmaking.

According to the Lewinian vision depicted in Figure 1 and itemized in Table 1, the approach to equilibrium of the forces is accelerated by "large-data stewardship and responding to evolving events in an agile manner ", [7]. In applications involving the use of corporate organizational theory [8] and systems engineering applications [9], it is also possible to demonstrate the three primary functions to manipulate and regulate the equilibrium state by i) facilitating, ii) constraining (with prioritized barrier levels) and iii) blocking data transfer and information flow to safeguard against dissemination of misinformation and incomplete and contradictory information used in field assessment. This approach also fits well with the management of ethical, legal, technical, commercial and quality issues associated with the acquisition, storage and sharing of individual data in a national and international large-data framework; see also [7]. All corporate industrial and professional organizations as well as local and regional authorities need to part of a coordinated to strategy effort to facilitate, constrain and blocking of data transfer in response to evolving scenarios of the spread of the Covid-19 pandemic. Only then the top-level decision-making will prove to be on target to deliver effective corrective actions to maintain the force-field balance.

Pharmaceutical and Medical Interventions and Clinical Research Methods

In an address to the nation, the German Chancellor hasindicated that if no non-pharmaceutical intervention is administered and the industrial and commercial activities are allowed to continue at the pre-pandemic levels, about $60 \%$ of the entire population of the country will be infected by Covid-19 with the virus reproduction number settling at the Ro $=2-3$ bracket [10]. A more recent Royal Society-British Academy Vaccine Report [11] evaluating possible strategies for vaccine deployment recognizes that up to $80 \%$ of the entire population may need to be vaccinated to provide effective community protection depending upon vaccine efficacy and the duration of protection (immunity provision) after first two (primary +booster) rounds of inoculation. Given these two high numbers of population immunization projected as a prerequisite to return to pre-pandemic levels of industrial and commercial activity and for the economies to function as normal, it is imperative that the non-pharmaceutical intervention measures listed in Table 1 to continue for some considerable amount of time whilst the population immunization is underway at mass scales. Accordingly, public expectations of "return to normality" need to be managed to allow for longer-term transition to normal whilst all the non-pharmaceutical interventions remain in place. Furthermore, if vaccine uptake remains optional, given the need for non-pharmaceutical restrictions and interventions to remain in place for a long time to come, human behavioral factors will come into play as impediment to the rate of mass population vaccination efforts. These behavioral factors include i) complacency based on diminished perception of high risk of fatality, ii) convenience and cost acting as barriers to accessibility, iii) trust and confidence in vaccine efficacy and safety, iv) lack of reliable sources of information on socio-demographic variation of immunization levels and challenges and uncertainties associated with vaccine deployment.

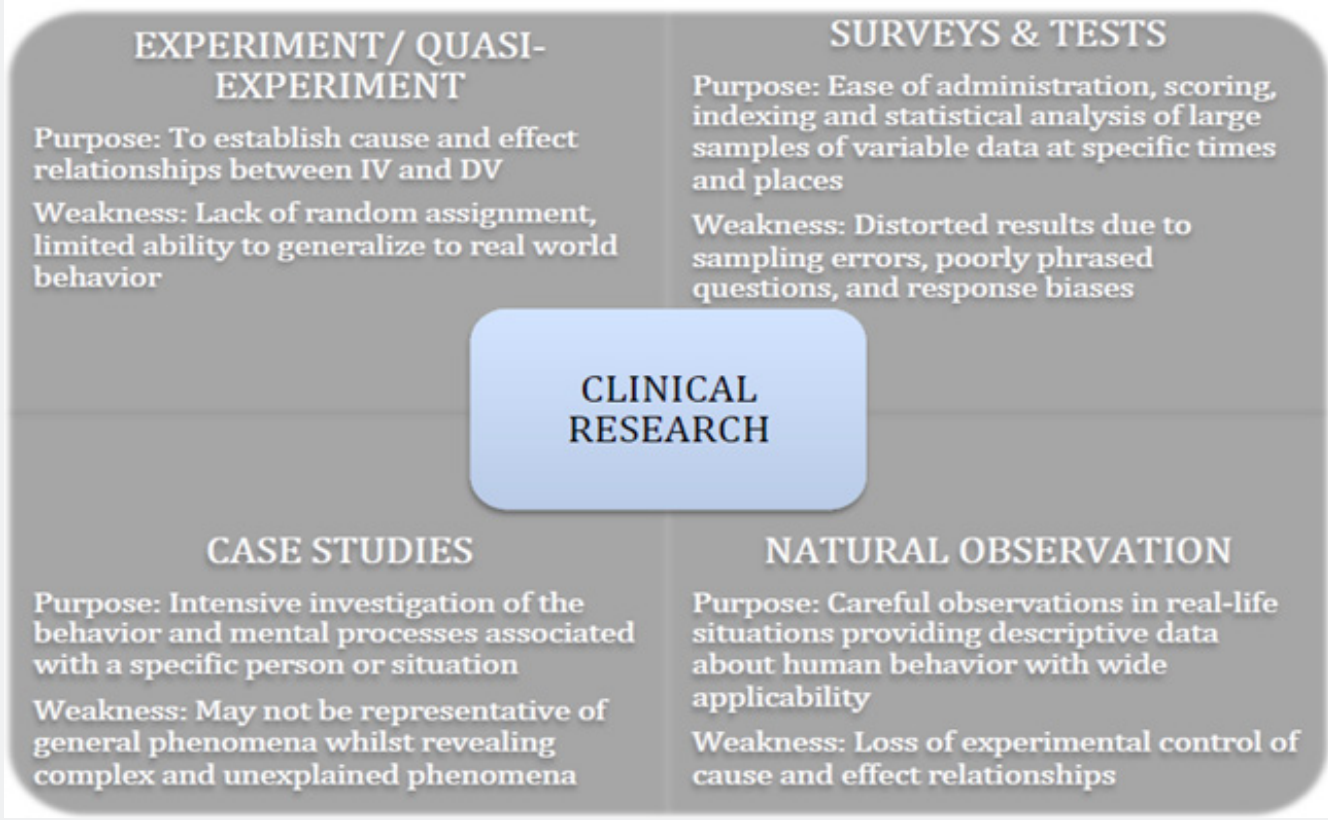

Figure 2: Clinical Research Methods Available for Research into the Physical, Psychological and Sociological Health and Wellbeing of Human Populations during the Covid-19 Pandemic. 
To address the above challenges brought on by human behavioral impediments to vaccine administration, the vaccine development efforts also need to be urgently accompanied by clinical research studies of i) effects on transmission dynamics of Covid-19 by administration of vaccines of varying micro-biological and clinical characteristics, ii) immune response and duration of immunity provision with different vaccines, iii) deployment priorities and demographic population structure prioritization for vaccination with different vaccine mixes of high and low cost and accessibility, iv) storage and transport facilities and conditions for different vaccines. Figure 2 shows the established clinical research methods with their respective purposes and weaknesses.

It is clear from Figure 2 that as the investigations are extended from controlled laboratory experiments involving ascertainment of relationships between key clinical dependent (DV) and independent (IV) variables, the uncertainties set in due to lack of control over cause and effect relationships in a multivariant demographic environment and errors introduced with sampling biases and lack of clarity of population responses. These errors and biases then reflect adversely in subsequent statistical analyses of large data sets resulting ultimately in potential misinterpretations of general phenomena. Furthermore, subsequent public admissions of errors in the clinical research methods and field trials could seriously undermine public confidence whilst also impacting adversely on the time schedules for the relaxing and eventual uplifting of restrictions on socio-economic activity. Hence, the early coordination of the field clinical research activities is an essential prerequisite to achieve the permanent containment of the spread of the virus regionally, nationally and globally. This is made possible only by the organized transfer of clinical knowledge and methodology to the field studies involving demographic science; refer also to Dowd et al. [6]. Transferring from clinical science to street science will allow for establishment of rules of human behavioral response with wide applicability in real-life situations.

Indirect Passive Forms of Pharmaceutical Intervention and Reliance on Uniform Human Immune System Response in a wide range of Environmental and $\mathrm{Hu}$ man Conditions

The author had refuted [3] the medical null hypothesis that prescribes one uniform form of vaccination application regime as 'once and for all' remedial measure to combat the Corona virus infections irrespective of real-life systems of human and environmental conditions. The primary support for this refuting argument comes from the very nature of the clinical vaccine developments to date. The major clinical developments of the Covid-19 vaccine build on the genomic sequencing of the virus DNA and then use this knowledge to synthetically construct human immune system alerts (e.g. in the form of messenger RNA, or as biochemically deactivated virus form) to familiarize the immune system with the genetic imprint of the virus in the expectation that the immune system will respond with boosted antibody production that will fight the live virus entering the bloodstream before the bloodstream infection becomes cell infection by attaching to and penetrating the cells. This form of indirect passive pharmaceutical intervention has the disadvantage of confirming efficacy and side-effect safety only indirectly through the beneficial human immune system response to be monitored over a prolonged period of time. With this form of indirect and passive pharmaceutical intervention, it will not be possible to determine the success rate of the vaccine applications instantly and conclusively.

Alternative methods possible that enable direct and active pharmaceutical intervention are possible by the development of either i) antibody boosters such as T-Cell production boosters or ii) virus neutralizers whose direct actions can be verified by routine Covid-19 antigen testing already in wide use to detect the infected individuals that test positive. Such rapid confirmation routes will not be possible with the current passive and indirect vaccine regimes that rely on healthy human immune system response over a period of time to confirm efficacy and safety of the vaccine composition and dose. Ferguson et al. [12], in their possible mitigation strategy developments to combat an influenza epidemic, also provide some worthwhile clues as to the distinctions between active and passive pharmaceutical intervention methods.

Moreover, the human immune system response will be affected by the presence of comorbidity due to the underlying health conditions. As such conditions will prevail to a much greater extent in the elderly population so will the variability of the intensity and duration of beneficial human immune system response. All of this makes it all the more important to advance the clinical research methods from analysis/synthesis laboratory experimentation for vaccine development to other large field-based methods illustrated in Figure 2 so that concurrent monitoring of the variability of the efficacy and the safety of the vaccines can be affected prior to/ during/post-administration to cross sections of the population. The rather scarce and sketchy reports of efficacy results of the current clinical vaccine trials already happen to confirm most of these hypotheses with more to emerge over a long period of transition to beneficial human immune system responses. Only then, will it be possible to go back to clinical analysis and synthesis of new vaccine formulations based on the findings of large fieldbased research methods. Hence, the so-called street science will decide the future directions of the clinical vaccine development for indirect passive pharmaceutical intervention over a prolonged period of transition.

\section{Conclusion}

In combatting the coronavirus epidemic, the nonpharmaceutical and indirect passive pharmaceutical intervention regimes currently enjoying world-wide support are critically evaluated in the context of a topological field theory of human 
behavioral responses and consequences first proposed by Kurt Lewin some several decades ago. It is possible to arrive at a first order connectivity mapping of the person and the environment versus behavior and consequences that alter the environment using time and space variations of all contributing factors. This approach lends itself well to holistic systems modelling of the forces driving and resisting the spread of COVID-19 pandemic balanced against the forces for societal change and measures applied to restrain the adverse effects. Furthermore, time and location dependent data sets can be used to demonstrate second order effects of modified social behavior patterns based on the altered states of infection risk perception in young and elderly age groups with different socioeconomic and professional backgrounds.

Large field-based research methods and acquisition and supervised utilization of large socio-economic and medical data sets are needed to enable decision-making in evolving emergency scenarios. These individual data are to be anonymized, aggregated and statistically calibrated to monitor the efficacy and the safety of the current indirect passive forms of vaccination. Stewardship of large data sets and organized and structured information flow are critical to the successful application of the topological field models of human behavioral responses to combat the spread of the Covid-19 pandemic. In early clinical trial results, the efficacy and safety of these indirect passive vaccination regimes are characterized significantly with the variability of intensity and duration of infection immunity provided in various population cross sections with or without underlying chronic health conditions.

It is recommended that further clinical therapeutic formulations also include direct and active pharmaceutical remedies that can stop the cell infection by the virus following the spread by the bloodstream infection. Earlier work with influenza pandemic mitigation strategies suggests strong clues in this direction.

\section{References}

1. Lewin K (1966) Principles of Topological Psychology (originally Gestalt Psychologie (1936)) translated by Heider F, Heider GM, McGraw-Hill Publ, New York, United States.

2. Lewin K (1943) Defining a Field at a Given Time. Psychological Review 50(3): 292-310.

3. Tuzun U (2020) COVID-19: How to Disprove the Medical "Null Hypothesis" by the Systems of Systems Approach to Operant Conditioning of Social Behavioral Response. Psychology and Behavioral Science International Journal 15(2): PBSIJ.MS.ID. 555907(2020).

4. Tuzun U (2020) Introduction to Systems Engineering and Sustainability Part I: Student-Centered Learning for Chemical and Biological Engineers, Education for Chemical Engineers, Elsevier, 31: 85-93.

5. Eichenbaum M, Godingo de Matos M, Lima F, Rebelo S Trabandt M (2020) How do People Respond to Small Probability Events with Large Negative Consequences?

6. Dowd JB, Andriano L, Brazel DM, Rotondi V, Block P, et al. (2020) Demographic Science in Understanding the Spread of Fatality Rates of COVID-19, PNAS (USA) 117(18): 9696-9698.

7. Royal Society DELVE Initiative, (2020) Data Readiness: Lessons from an Emergency, DELVE Report no: 7, 20 November 2020

8. Cronshaw SF., McCulloch ANA (2008) Reinstating the Lewinian Vision: From Force Field Analysis to Organization Field Assessment, Organization Development Journal, Chesterland 26(4): 89-103.

9. Smartt C, Casey W, Ferreira S (2018) Using Force-Field Analysis as Part of Systems Engineering Strategy to Achieve Goals, INCOSE International Symposium, 28(1): 1420-1432, Wiley Online Library.

10. Merkel A (2020) Merkel Gives Germans a Hard Truth about the Coronavirus, New York Times, United States.

11. Royal Society \& British Academy (2020) COVID-19 Vaccine Deployment: Behavior, Ethics, Misinformation, and Policy Strategies, Review Report pre-print submitted to peer-review, 21 October 2020, Leverhulme Centre for Demographic Science, University of Oxford, DES7210, Royal Society, UK.

12. Ferguson NM, Cummings DAT, Fraser C, Cajka CJ, et al., (2006) Strategies for Mitigating an Influenza Pandemic, Nature 442(7101): 448-452.

\section{Your next submission with Juniper Publishers} will reach you the below assets

- Quality Editorial service

- Swift Peer Review

- Reprints availability

- E-prints Service

- Manuscript Podcast for convenient understanding

- Global attainment for your research

- Manuscript accessibility in different formats

( Pdf, E-pub, Full Text, Audio)

- Unceasing customer service

Track the below URL for one-step submission https://juniperpublishers.com/online-submission.php 